\title{
Inbuilt tendency of the eIF2 regulatory system to counteract uncertainties
}

\author{
Mohammad Farhan Khan*, Sarah K. Spurgeon ${ }^{\dagger}$, Xing-Gang Yan $^{\S}$, Muaffaq M. Nofal ${ }^{\ddagger}$, Rami Al-Hmouz
}

\begin{abstract}
Eukaryotic initiation factor 2 (eIF2) plays a fundamental role in the regulation of protein synthesis. Investigations have revealed that the regulation of eIF2 is robust against intrinsic uncertainties and is able to efficiently counteract them. The robustness properties of the eIF2 pathway against intrinsic disturbances is also well known. However the reasons for this ability to counteract stresses is less well understood. In this paper, the robustness conferring properties of the eIF2 dependent regulatory system is explored with the help of a mathematical model. The novelty of the work presented in this paper lies in articulating the possible reason behind the inbuilt robustness of the highly engineered eIF2 system against intrinsic perturbations. Our investigations reveal that the robust nature of the eIF2 pathway may originate from the existence of an attractive natural sliding surface within the system satisfying reaching and sliding conditions that are well established in the domain of control engineering.
\end{abstract}

Index Terms-Protein synthesis, mathematical modelling, linearisation, key non-linearities, stability, sliding surface.

\section{INTRODUCTION}

The regulation of gene expression takes place at multiple levels. However it is primarily controlled by the machinery of translation initiation [1]-[3]. There are various eukaryotic initiation factors (eIFs) that play a crucial role in modulating the dynamic control properties of translation initiation. Eukaryotic initiation factor 2 (eIF2) is one of the main factors that sustain the ongoing translation activity [4], [5]. Defects or down-regulation in eIF2 can result in severe illnesses, for example, disturbance in the translation initiation machinery due to excessive phosphorylation of eIF2 can cause neurological diseases [6]-[8].

The perpetuation of sustainable behaviour of the translation activity is achievable only when eIF2 regulates between its active (GTP-bound) and inactive (GDP-bound) states, and carries out its fundamental role of transferring Met-tRNAi (initiator transfer ribonucleic acid) to the $40 \mathrm{~S}$ ribosomal subunit without interruption. However, during amino-acid starvation, phosphorylation of the $\alpha$-subunit of eIF2 takes place, which results in down-regulation of general translation activity [9], [10].

${ }^{*}$ M.F. Khan is with Department of Electrical Engineering, Indian Institute of Technology Roorkee, Roorkee - 247667, India (Corresponding author email: farhan7787@gmail.com)

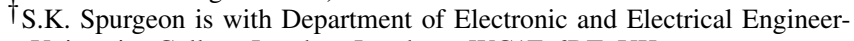
ing, University College London, London - WC1E 6BT, UK

\& X. Yan is with School of Engineering and Digital Arts, University of Kent, Canterbury - CT2 7NT, UK

$\mp$ Department of Mathematics and General Sciences, Prince Sultan University, Riyadh - 11586, KSA

Department of Electrical and Computer Engineering, King Abdulaziz University, Jeddah - 21589, KSA
The phosphorylation process is one of the main disruptors of translation activity, converting eIF2 to a dominant inhibitor of the guanine nucleotide exchange factor (GEF) eIF2B, which disrupts the upcoming round of translation activity. The phosphorylated eIF2 loses the affinity with eIF2B or converts from a substrate to an inhibitor of eIF2B, and hence is unable to re-energise its active state with the help of eIF2B, resulting in disruption of the translation process [11], [12].

There are four important kinases that have a tendency to phosphorylate the eIF $2 \alpha$, namely general control nonderepressible-2 (GCN2), protein kinase double-stranded RNAdependent (PKR), PKR-like ER kinase (PERK), and hemeregulated inhibitor (HRI) [13]. From the kinases, GCN2 is one of the most highly conserved that activates due to the presence of uncharged tRNA and primarily targets eIF2 [14], [15]. Hence, the kinase GCN2 is a focus in this study and is included in the mathematical model to understand the dynamic aspects of the eIF2 dependent regulatory pathway. The regulatory pathway representing the impact of phosphorylated eIF2 (eIF2-P) on translation activity or protein synthesis is illustrated in Fig. 1. The figure demonstrates eIF2-P as a competitive inhibitor of eIF2B in the form of bold black dots.

Investigations have revealed that the eIF2 pathway is robust against uncertainties and can counteract disturbances created by intrinsic stressors [16], [17]. The robustness properties of biomolecular processes against internal and structural disturbances are well known. However the reasons for this ability to cope against stresses is less well understood [18]-[20]. Hence, this study is focused on investigating the possible reason behind the inbuilt tendency of the highly engineered eIF2 dependent system to counteract intrinsic uncertainties.

In control theory, the application of sliding mode control has been widely acknowledged to efficiently stabilise the uncertain nonlinear systems, such as power systems, biped robots etcetera [21]. Analogous to the highly engineered system, most of the biological systems naturally hold the high level of robustness against disturbances, possibly due to the existence of natural sliding surface within the system which is able to attain the stable sliding motion by reaching the phase where the motion converges from a non-zero initial value to the naturally existing sliding surface and then converges to the origin along that sliding surface.

The remainder of the paper is organized as follows. In Section II, a mathematical model of eIF2 regulatory system is presented. Section III presents the limitations of the linearisation concept of the nonlinear eIF2 model. The role of key nonlinearities in maintaining the temporal behaviour of the model is explored in Section IV. The same section also 


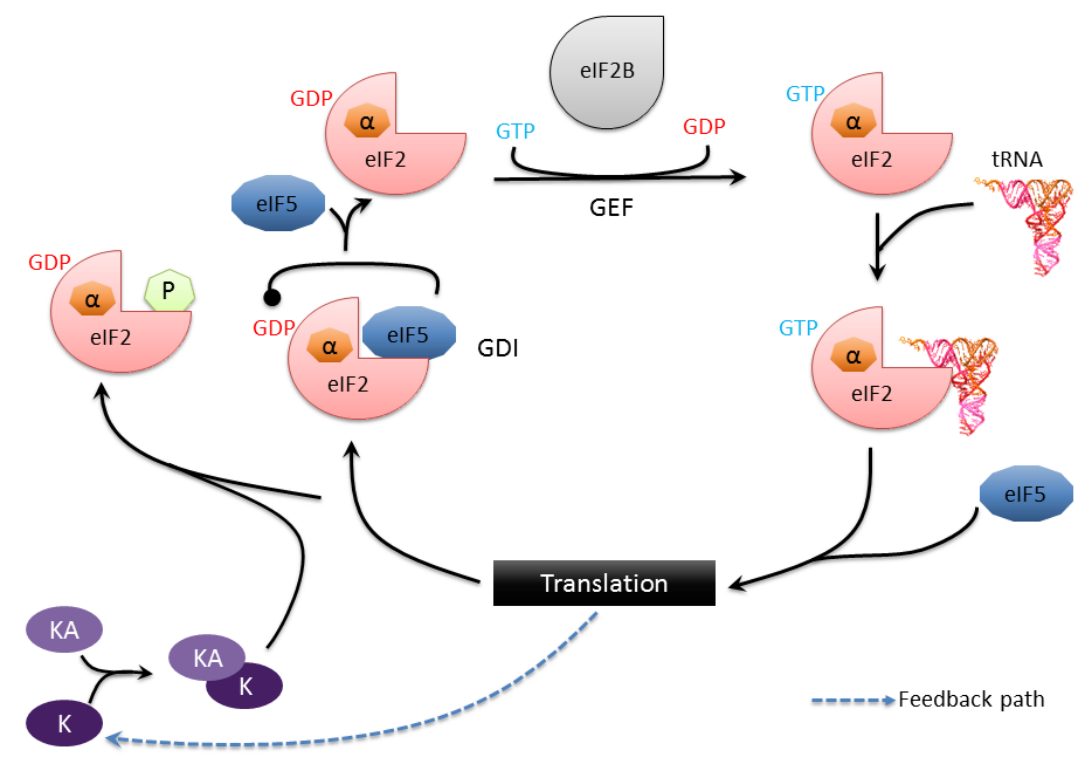

Fig. 1. Impact of phosphorylation of eIF2 on translation initiation machinery. The eIF5 has an additional role of GDP dissociation inhibitor (GDI) in translation initiation along with its established functions in pre-initiation complex assembly and GTPase activating proteins (GAPs) activity. The kinase K (GCN2) is activated by activator KA (uncharged tRNAs) and forming a feedback path for its regulation

investigates the robustness conferring nature of eIF2 pathway against intrinsic stresses. Section $\mathrm{V}$ presents the novel theory regarding the possible reason behind the inbuilt tendency of the highly engineered eIF2 system to counteract intrinsic uncertainties. Finally, the paper is concluded in Section VI.

\section{MATERials AND METHODS}

Molecular reaction network theory is one of the earliest attempts to theoretically model the dynamical behaviour of biomolecular systems. Reaction network theory has also attracted researchers from other communities where nonlinear dynamical systems are examined such as biochemistry, physics, control engineering and theoretical chemistry [22][25]. The reaction networks resembling the nonlinear interaction of a large number of biological elements are very complex in nature [26], [27]. Therefore some model reduction transformations or simplifications have been suggested in the literature to reduce the complexity of large reaction networks. Examples include linearisation of the resulting network, model reduction based on quasi steady state analysis and gramianbased input/output balancing [28]-[30]. It should be noted that each species of the regulatory pathway should hold a positivity characteristic during any simplification or reduction process. During simulation the concentration of each species (say $Y$ ) must satisfy the following proposition.

Proposition: The concentrations $Y_{i} \forall i \in[1, n]$ of all $n$ species in a given model have non-negative values, that is $Y_{i}$ $\geq 0, \forall t \geq 0$.

One of the well known approaches to efficiently model regulatory systems is to focus on the details of the core reactions under study [31]-[34]. In addition, it is desirable to combine the theoretical model with experimental data. In the present study, the core reactions constituting eIF2:GDP complexes (refer Fig. 1) are considered for developing a mathematical model, which is comprised of two stages, namely the unstressed and stressed stages. The unstressed stage is the one in which kinase GCN2 is not active and the translation machinery is uninterrupted. The stressed stage is the one in which phosphorylation of eIF2 takes place due to activation of GCN2, resulting into cease in the translation activity. The overall reaction model of the eIF2 regulatory system is as follows:

$$
\begin{aligned}
& \text { [Protein] } \stackrel{C_{1}}{\rightarrow} \text { [eIF5:eIF2:GDP] } \\
& \text { [eIF5:eIF2:GDP] }+\left[\text { eIF2B] } \underset{C_{3}}{\stackrel{C_{2}}{\rightleftharpoons}}[\text { eIF5:eIF2B:eIF2:GDP] }\right. \\
& \text { [eIF5:eIF2B:eIF2:GDP] } \underset{C_{5}}{\stackrel{C_{4}}{\rightleftharpoons}}[\text { eIF5] }+[\text { eIF2B:eIF2:GDP] } \\
& \text { [eIF5:eIF2:GDP] } \underset{C_{7}}{\stackrel{C_{6}}{\rightleftharpoons}}[\text { eIF2:GDP] }+[\text { eIF5] } \\
& {\left[\text { eIF2B] }+\left[\text { eIF2:GDP] } \underset{C_{9}}{\stackrel{C_{8}}{\rightleftharpoons}}[\text { eIF2B:eIF2:GDP] }\right.\right.} \\
& \text { [eIF2B:eIF2:GDP] } \stackrel{C_{10}}{\rightarrow}[\mathrm{eIF} 2 \mathrm{~B}]+[\mathrm{eIF} 2: \mathrm{GTP}] \\
& {\left[\text { eIF5] }+\left[\text { eIF2:GTP] } \stackrel{C_{1}}{\rightarrow}\right. \text { [Protein] }\right.} \\
& {[\mathrm{GCN} 2]+[\mathrm{tRNA}] \underset{\mathrm{C}_{13}}{\stackrel{C_{12}}{\rightleftharpoons}}[\mathrm{tRNA}: \mathrm{GCN} 2]} \\
& \text { [eIF2:GDP] + [tRNA:GCN2] } \underset{C_{15}}{\stackrel{C_{14}}{\rightleftharpoons}} \text { [tRNA:GCN2:eIF2] } \\
& \text { [eIF2-P] }+\left[\text { eIF2B] } \underset{C_{17}}{\stackrel{C_{16}}{\rightleftharpoons}}[\text { eIF2-P:eIF2B] }\right. \\
& \text { [eIF2-P] } \stackrel{C_{18}}{\rightarrow} \text { [eIF2:GDP] } \\
& \text { [tRNA:GCN2:eIF2] } \stackrel{C_{19}}{\rightarrow} \text { [tRNA:GCN2] + [eIF2-P] } \\
& \text { [Protein] } \stackrel{C_{20}}{\rightarrow}[\text { tRNA] }
\end{aligned}
$$

The detailed nonlinear ordinary differential equations (ODEs) of the overall reaction model, initial concentrations and rate constant values are described in supplementary files $\mathrm{S} 1, \mathrm{~S} 2$ and S3 respectively. 
(a)

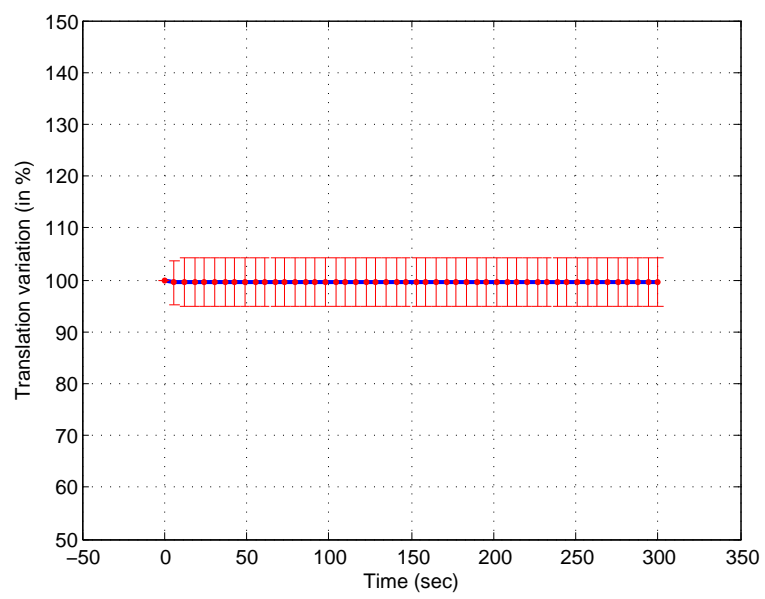

(b)

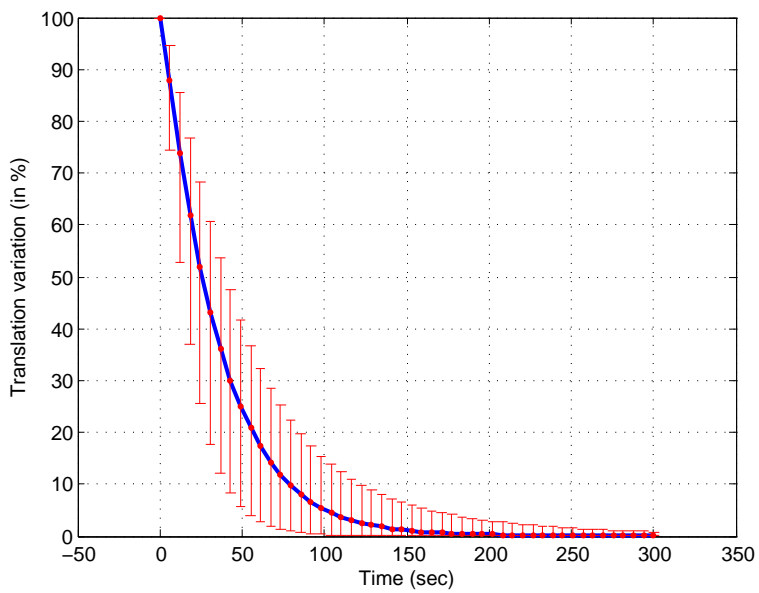

Fig. 2. Impact of mutation induced changes in the rate constants of the pathway for: (a) unstressed stage, and (b) stressed stage, resulted into controlled variation in the overall translation activity from its experimental value. The blue line represents mean behaviour of the eIF 2 model for the rate constants described in supplementary file S3, while red error bar represents the deviation of the model from its mean behaviour due to perturbations in the rate constants.

The experimental observations of eIF2 pathway have revealed that the translation activity of the pathway illustrated in Fig. 1 is insensitive to the changes in the levels of eIF2, eIF2B and eIF5 [4], and is also robust against the mutation induced changes in the rate constants [35]. That is, the tolerance characteristic of the eIF2 pathway against intrinsic robustness is naturally controlled might be due to the fact that the pathway is structured in such a way that its output is relatively insensitive to the variations in the micro-environment [36]. Computationally such intrinsic robustness can be visualised by randomly perturbing the rate constants of the model to the limit of $\pm 50 \%$ form its actual values described in supplementary file S3.

Fig. 2 summarises the impact of intrinsic perturbations in the eIF2 pathway for unstressed and stressed stages due to mutation induced changes in the rate constants, which are accordant with the biological observations. The robustness conferring property of the eIF2 pathway against disturbances is well known, however the reasons for this ability is less well understood. From the control theory perspective, it can be proposed that the natural phenomena of intrinsic robustness against perturbations in both the stages is likely due to the preexistence of a highly engineered natural control within the eIF2 pathway which is helping the system to cope with such uncertainties.

\section{LINEAR APPROXIMATION OF NONLINEAR EIF2 SYSTEM}

In order to investigate the ability of the highly engineered eIF2 system to counteract uncertainties, it is necessary to first simplify or linearise the developed nonlinear ODE model. Linear models can be efficiently analysed in the frequency domain which can predict the non-trivial states of the system that are responsible for maintaining its basal activity [17]. The linearisation process is helpful in approximating the higher order nonlinear system by a lower order linear system, through which the local behaviour of nonlinear systems can be estimated around the equilibrium point [37]-[39]. The feasible equilibrium point of the eIF2 pathway is given in supplementary file S4.

The generalised form of the nonlinear mathematical model described in supplementary file S1 can be defined as follows:

$$
\dot{Y}(t)=f(Y, t)
$$

where, $Y$ is the non-negative concentration of the species and $t$ is the evolution time. The state space representation of the approximate linear model around equilibrium point $Y^{e q}$ using the Jacobian matrix $A$ of the vector $f(\cdot)$ can be re-written in the form:

$$
\dot{Y}(t)=A Y(t)
$$

The diagonal elements of $A$ (presented in the supplementary file S5) represent the behaviour of the species due to reversible (or irreversible) interaction, whereas non-diagonal elements represent interactions between species. In order to compare the performance of the linear and nonlinear models, $Y^{e q}$ is considered as an initial condition of the nonlinear model, which has been perturbed by a small amount $\delta$ and then the same $\delta$ value is used as an initial condition of the corresponding linear model.

The comparison of steady states of both models for different values of $\delta$ is given in supplementary file S6. Observe that as the value of $\delta$ increases, the difference between the models becomes substantial.

In order to investigate the origins of robustness within the system with the help of applied control theory, the overall system is converted into a single input single output (SISO) system by eliminating the dynamics of uncharged tRNA and considering it as a control input $u$ [17].

Consider the nonlinear system described in supplementary file S1 where the dynamics of uncharged tRNA has been eliminated. The generalised state space representation of the system can be written in the following SISO form:

$$
\begin{aligned}
\dot{Y}(t) & =\mathbb{Y}(Y, t)+\mathbb{B}(Y(t)) u \\
Z(t) & =Y_{1}(t)
\end{aligned}
$$




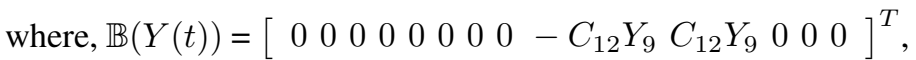
$Z(t)$ is the output signal or translation rate, and vector $Y=\left[\begin{array}{lllll}Y_{1} & Y_{2} & \cdots & Y_{12} & Y_{14}\end{array}\right]^{T}$.

Investigations illustrated in supplementary file S7 have revealed that linearising the nonlinear SISO model in order to analyse the trivial species in the eIF2 pathway using the matched DC gain method [40] produces unfeasible temporal behaviour such as a substantial difference in the transient behaviour of models and concentrations of kinase and kinaseactivator reaching negative values at certain physiological time instants. In order to cope with the problem of the infeasible behaviour of the system, the key nonlinearities of the system have been estimated and restored. This is necessary to preserve the overall transient behaviour of the system, and hence the approximate model can be used to estimate the origins of robustness within the highly engineered eIF2 dependent system that are responsible for counteracting the intrinsic uncertainties.

\section{RESTORING KEY NONLINEARITIES WITHIN THE EIF2} SYSTEM

As stated earlier, linearisation is a helpful tool in simplifying the nonlinear interactions between the species, so that the individual effect of each parameter can be investigated. However linearising the whole system may produce an unacceptable transient response. Therefore in this section emphasis is given to illustrating the role key nonlinearities play in maintaining the temporal behaviour of the system.

Consider a SISO system defined in eq.(3), which can be rewritten in the generalised state space form:

$$
\begin{aligned}
\dot{Y}(t) & =\hat{Y}(Y, t)+\varphi(Y, t)+\mathbb{B}(Y(t)) u \\
Z(t) & =Y_{1}(t)
\end{aligned}
$$

where, the term $\varphi(Y, t)$ represents important nonlinearities of the system. Note that, $\mathbb{Y}(Y, t)=\hat{\mathbb{Y}}(Y, t)+\varphi(Y, t)$. Now, linearising the whole system expect $\varphi(Y, t)$ around an equilibrium point will result in the partially linearised SISO model given in supplementary file S8. The generalised state space system of the model can be defined as:

$$
\begin{aligned}
\dot{Y} & =\hat{A} Y+\varphi(Y, t)+B u \\
Z & =D Y
\end{aligned}
$$

where $\hat{A}$ is a Jacobian matrix, $B$ and $D$ are constant matrices, and $\varphi(Y, t)$ is a nonlinear vector defined as:

$$
\varphi(Y, t)=\left[\begin{array}{lllllllllllll}
0 & 0 & -\gamma & 0 & 0 & 0 & 0 & 0 & 0 & 0 & -\gamma & \gamma & 0
\end{array}\right]^{T}
$$

where, $\gamma=C_{16} Y_{3} Y_{11}$. The above system which includes key nonlinearity is expected to provide better temporal behaviour than a completely linearised system when compared with the original nonlinear system. The figures in supplementary file S9 illustrate the important role of key nonlinearities within the eIF2 system in maintaining the temporal behaviour of the model. It is worth noting that the partially linearised SISO system should not only possess approximate temporal behaviour but also exhibit similar stability properties to those exhibited by the nonlinear SISO system.
Consider a candidate Lyapunov function $V(Y, t)$ for the eIF2 system defined in supplementary file S8:

$$
V(Y, t)=\sum_{i=1}^{14} Y_{i}+Y_{1}+Y_{2}+2 Y_{4}+Y_{6}+Y_{10}+2 Y_{14}
$$

where $V(0, t)=0$ and $V(Y, t)>0, \forall Y \neq 0$.

According to Lyapunov theory, the system is stable if the temporal derivative of $V(Y, t) \leq 0$. Following the Lyapunov stability theorem, the eIF2 system is said to be stable if $\dot{V}(Y, t)$ for the eIF2 system is negative semidefinite. The temporal derivative of eq.(6) can be defined as:

$$
\begin{aligned}
\dot{V}(Y, t) & =\sum_{i=1}^{14} \dot{Y}_{i}+\dot{Y}_{1}+\dot{Y}_{2}+2 \dot{Y}_{4}+\dot{Y}_{6}+\dot{Y}_{10}+2 \dot{Y}_{14} \\
& =-\left(C_{16} Y_{3} Y_{11}+C_{17} Y_{12}+C_{20} Y_{1}\right)
\end{aligned}
$$

Since $C_{16}, C_{17}$ and $C_{20}$ are non-negative rate constants, from eq.(8) it is evident that $\dot{V}(Y, t)$ is negative semidefinite. Therefore the approximate eIF2 system defined in supplementary file S8 is stable. Further, it can be observed that perturbing the rate constants will have no impact on the overall stability of the dynamical system. This observation leads to the conclusion that the eIF2 pathway is a highly engineered system that can efficiently tolerate intrinsic perturbations without compromising the overall stability characteristics.

\section{EXISTENCE OF NATURAL SLIDING SURFACES WITHIN THE EIF2 REGULATORY MODEL}

In control theory, model uncertainties can be divided into two forms, namely matched and unmatched uncertainties. Generally, in a given dynamical system if uncertainty lies in the input channel, then this type of uncertainty is known as matched uncertainty. On the other hand, unmatched uncertainty corresponds to uncertainty acting in channels that are not implicit in the input channels. Note that, in engineering systems not all unmatched uncertainty can be rejected by design of a control but an appropriate control will reject matched uncertainty completely [21].

The motivation for considering this theory is to provide evidence of robust conferring feature in the biomolecular systems against intrinsic disturbances. That is, the biomolecular systems are robust and relatively insensitive to alterations in their internal parameters and are able to adapt to changes in their micro-environment. In this section, matched uncertainties are embedded in the eIF2 system to investigate the reason behind the inbuilt tendency of the eIF2 regulatory system to counteract uncertainties. The supplementary file S10 defines the mathematical model of an eIF2 regulatory pathway with key nonlinearities and matched uncertainties.

Fig. 3 shows the inbuilt ability of the eIF 2 dependent regulatory model, which includes key nonlinearities, to counteract matched uncertainties. In other words, the pathway is able to completely reject matched uncertainty. It is observed from Fig. 3 that perturbing the value of $C_{13}$ to $100 \%$ from its reference value has no effect on the translational behaviour (red solid line) with respect to the behaviour of the SISO model including key nonlinearities (green dotted line). Fig. 4 
(a)

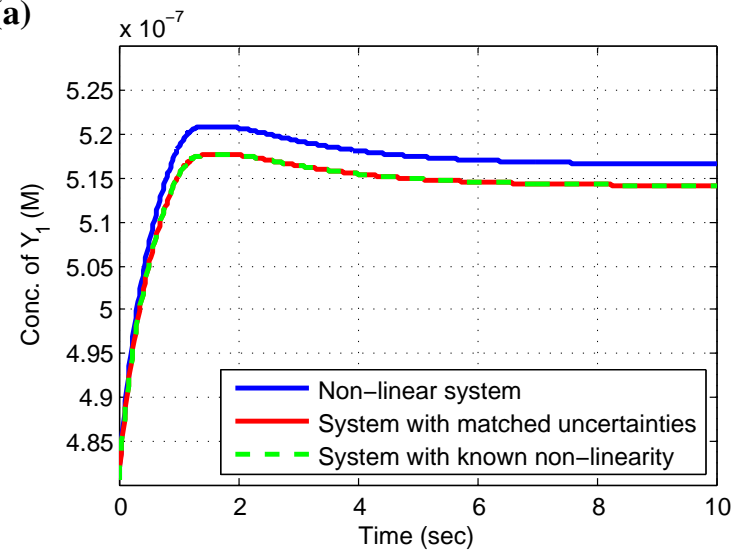

(b)

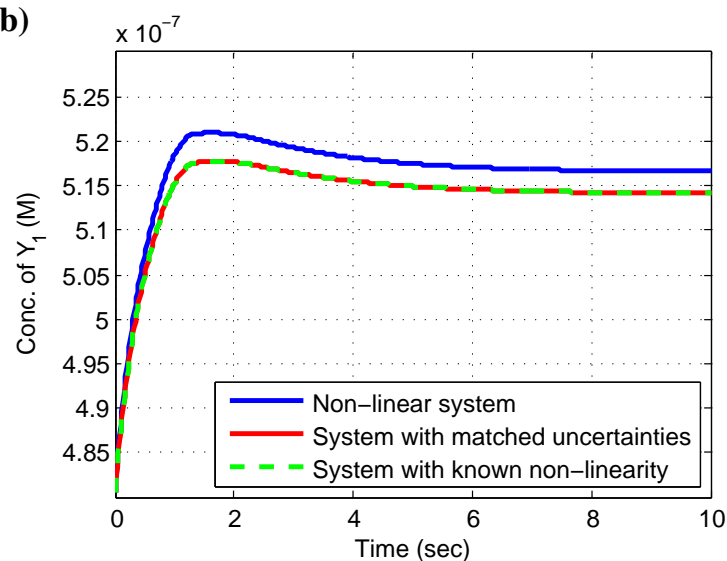

Fig. 3. Comparison of translation rate of nominal model, model with known nonlinearity and uncertain model with known nonlinearity around equilibrium point for: (a) $\delta=10^{-1}$ and $\Delta C_{13}=10^{+2} \times C_{13}$, and (b) $\delta=10^{-1}$ and $\Delta C_{13}=10^{-1} \times C_{13}$

(a)

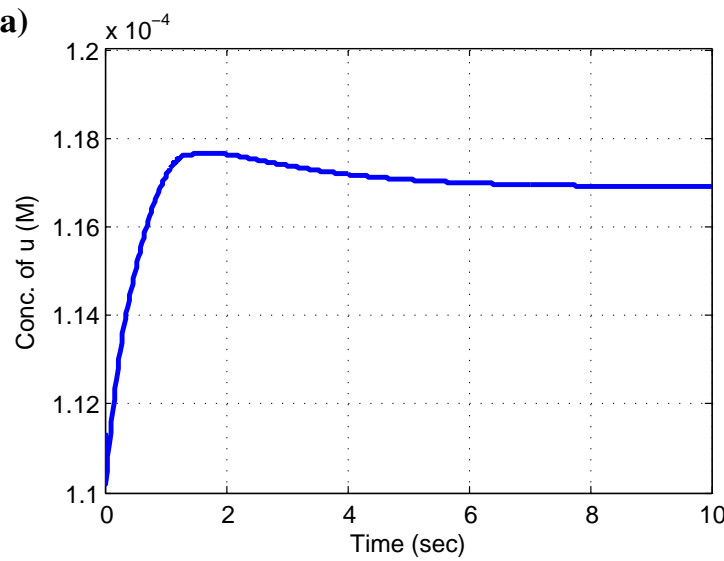

(b)

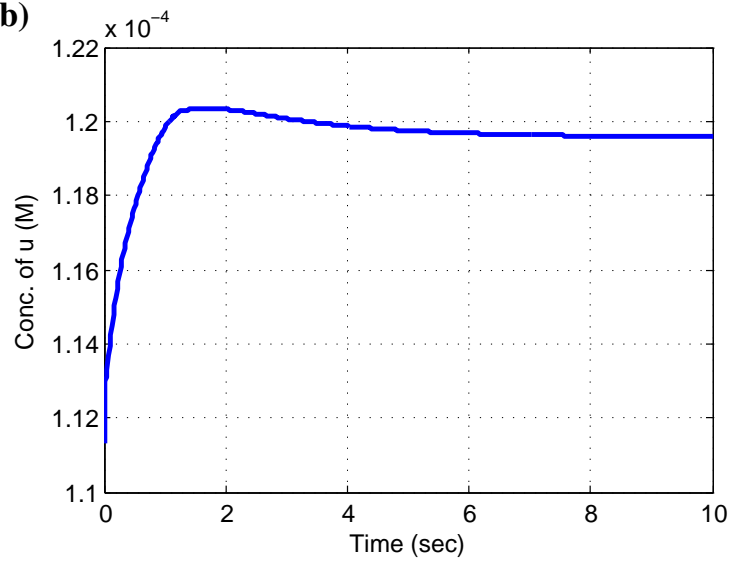

Fig. 4. Change in control $u$ for: (a) $\delta=10^{-1}$ and $\Delta C_{13}=10^{+2} \times C_{13}$, and (b) $\delta=10^{-1}$ and $\Delta C_{13}=10^{-1} \times C_{13}$

shows the time evolution of the control $u$ that is counteracting the effect of matched uncertainty in the eIF2 regulatory system.

The above observation asserts that the eIF2 dependent regulatory model is able to counteract matched uncertainties by natural control and making it robust against such uncertainties. One of the reasons behind such tolerance may be the existence of attractive natural sliding surfaces within the system which prescribe an appropriate stable sliding motion. The regulation within the system would effectively ensure a corresponding reachability condition is satisfied, which effectively ensures the sliding motion is established. In sliding mode control, motion of the system is divided into two phases: the first phase is generally called the reaching phase where motion converges from a non-zero initial value to the sliding surface, and the second phase of the motion is called the sliding phase during which the system converges to the origin along the sliding surface. The reachability condition accommodates the nonlinearities within the system and robustness properties with respect to parameter and modelling uncertainty are exhibited. Hence, the reachability analysis provides a dynamical condition for the translation response to reach and maintain the steady state. Satisfaction of both conditions ensures that system output is not affected by matched uncertainty and indicates the system includes a natural sliding surface which results in an ability to counteract uncertainty.

In order to verify the validity of this theory within the nonlinear system, consider the following nonlinear sliding surface for the eIF2 dependent regulatory model that has a relative degree of two.

$$
S=Y_{1} Y_{14}
$$

A nonlinear sliding function is chosen to fulfil both sliding and reaching conditions which is essential for counteracting matched uncertainties within the eIF2 dependent regulatory system. Note that the existence of a unique nonlinear sliding surface for both the non-stress and stress cases assures accomplishment of sliding motion and instigation of the natural tendency for robustness. In order to investigate the reachability condition, the derivative of the sliding surface is computed as follows:

$$
\begin{aligned}
\dot{S}= & \dot{Y}_{1} Y_{14}+Y_{1} \dot{Y}_{14} \\
= & -\left(C_{1}+C_{20}+C_{15}+C_{19}\right) Y_{1} Y_{14}+C_{11} Y_{5} Y_{8} Y_{14} \\
& +C_{14} Y_{7} Y_{10} Y_{1}
\end{aligned}
$$



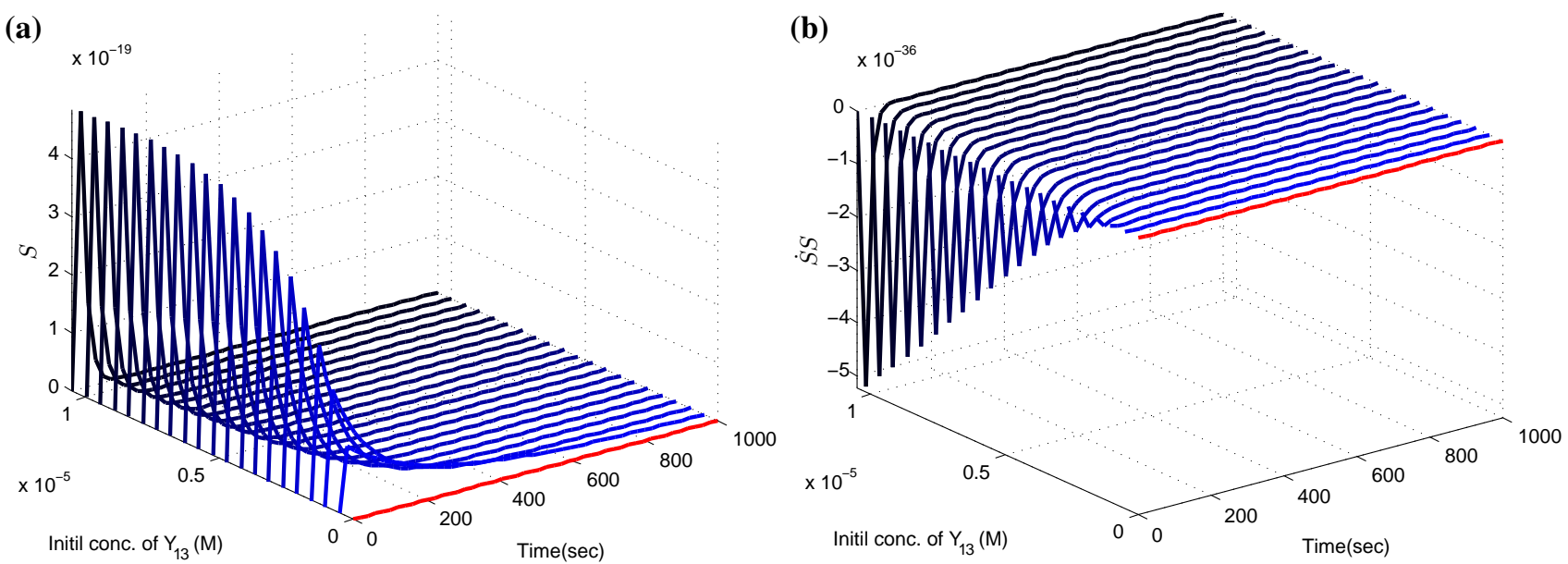

Fig. 5. Time evolution of: (a) sliding surface $(S)$, and (b) $\dot{S} S$ for varying concentration of uncharged tRNA

Considering both cases in the eIF2 pathway, the eq.(11) becomes:

Case I: Unstressed condition

Under nominal or unstressed condition, the accumulation of uncharged tRNA remains nil, which fails to induce any stress complexes into the nominal system, that is complexes such as tRNA:GCN2 $\left(Y_{10}\right)$, tRNA:GCN2:eIF2 $\left(Y_{14}\right)$ etcetera remain in an inactive state resulting in zero concentration.

Case II: Stressed condition

In the stressed condition, when the stress dynamics enters the eIF2 system, the uncharged tRNA starts accumulating which induces the stress complexes into the system resulting into cessation of protein synthesis $\left(Y_{1}\right)$ due to its dependency on core eIF2 complexes such as eIF2:GDP, eIF2:GTP $\left(Y_{8}\right)$ etcetera.

Considering the above cases, it can be seen that when the system moves from non-stress to stress condition or vice-versa, the temporal derivative of $S$ reaches zero. Fig. 5 illustrates the transient response of the sliding surface for varying concentration of uncharged tRNA. When the reachability condition is fulfilled, it follows that $S=\dot{S}=0$. Observing Fig. 5, it is evident that in the unstressed case the motion is strictly taking place on the constraint manifold, i.e. $S=0 \forall t$ and $\dot{S} S=0 \forall t$. Hence it can be established that in both unstressed and stressed cases, the sliding phase and reaching phase have been fulfilled i.e. the function $S$ reaches zero at finite time $t$ and $\dot{S} S \leq 0$.

Note that, in the scenario of biomolecular systems, satisfaction of the reachability condition gives rise to two distinct cases:

Case I: When $Y_{1}=0, Y_{14} \neq 0$

$\dot{S}=Y_{1} \dot{Y}_{14}+\dot{Y}_{1} Y_{14}=0$

$\Rightarrow \dot{Y}_{1} Y_{14}=0 \Rightarrow \dot{Y}_{1}=0$

$\Rightarrow-C_{1} Y_{1}+C_{11} Y_{5} Y_{8}-C_{20} Y_{1}=0$

$\Rightarrow C_{11} Y_{5} Y_{8}=0$

The above case is biologically infeasible because in the unstressed case $C_{11} Y_{5} Y_{8} \neq 0$.
Case II: When If $Y_{1} \neq 0, Y_{14}=0$

$$
\begin{aligned}
& \dot{S}=Y_{1} \dot{Y}_{14}+\dot{Y}_{1} Y_{14}=0 \\
& \Rightarrow Y_{1} \dot{Y}_{14}=0 \Rightarrow \dot{Y}_{14}=0 \\
& \Rightarrow C_{14} Y_{7} Y_{10}=0
\end{aligned}
$$

The aforementioned equality is biologically feasible under both stressed and unstressed cases, because in the unstressed case the concentration of tRNA:GCN2 $\left(Y_{10}\right)$ is zero while the concentration of eIF2:GDP $\left(Y_{7}\right)$ is nonzero, and as the stress dynamics enters the concentration of eIF2:GDP falls to zero while the concentration of tRNA:GCN2 increases.

Observing Case II, it can be asserted that when the reachability condition for a sliding mode is satisfied, the dynamics of the system self regulates itself and the system is driven towards a naturally existing sliding manifold and remains on it.

\section{CONCLUSION}

In this paper the inbuilt robustness tendency of the eIF2 dependent regulatory model has been explored with the help of a mathematical model and applied control theory. In order to explore the origins of the robustness conferring properties of the eIF2 system, a linearsation concept has been adopted. The study indicates that entirely linearising the eIF2 system around an equilibrium point results in unacceptable system behaviour. Therefore a key nonlinearity within the eIF2 system has been identified and restored within the model. A simplified mathematical model results which exhibits comparatively closer temporal behaviour to that of the original nonlinear system. The investigations have revealed that the eIF2 pathway is robust to intrinsic uncertainties and is able to efficiently counteract them without affecting system stability. One of the possible reasons behind this tendency of highly engineered eIF2 system to exhibit robustness against intrinsic perturbations is the existence of an attractive natural sliding surface within the eIF2 system that satisfies reaching and sliding conditions through which the system is able to counteract efficiently the disturbances. 


\section{REFERENCES}

[1] N. Sonenberg, and A. G. Hinnebusch, "Regulation of translation initiation in eukaryotes: Mechanisms and biological targets", Cell, vol. 136, no. 4, pp. 731-745, Feb. 2009.

[2] F. Gebauer, and M. W. Hentze, "Molecular mechanisms of translational control", Nature Reviews Molecular Cell Biology, vol. 5, no. 10, pp. 827-835, Oct. 2004.

[3] T. von der Haar, and J. E. G. McCarthy, "Intracellular translation initiation factor levels in Saccharomyces cerevisiae and their role in capcomplex function", Molecular Microbiology, vol. 46, no. 2, pp. 531-544, Oct. 2002

[4] H. Firczuk, S. Kannambath, J. Pahle, A. Claydon, R. Beynon, J. Duncan, H. Westerhoff, P. Mendes, and J. E. McCarthy, "An in vivo control map for the eukaryotic mRNA translation machinery", Molecular Systems Biology, vol. 9, no. 1, pp. 635-635, Apr. 2013

[5] M. F. Khan, S. K. Spurgeon, M. M. Nofal, and X. G. Yan, "SemiDisparate impact of kinases GCN2 and PERK in modulating the dynamic control properties of eIF2 pathway", IEEE Access, vol. 7, pp. 68132-68139, 2019

[6] T. Ma, M. A. Trinh, A. J. Wexler, C. Bourbon, E. Gatti, P. Pierre, D. R. Cavener, and E. Klann, "Suppression of eIF2 $\alpha$ kinases alleviates Alzheimers diseaserelated plasticity and memory deficits", Nature Neuroscience, vol. 16, no. 9, pp. 1299-1305, Aug. 2013.

[7] E. Mutez, A. Nkiliza, K. Belarbi, A. de Broucker, C. VanbesienMailliot, S. Bleuse, A. Duflot, T. Comptdaer, P. Semaille, R. Blervaque, D. Hot, F. Leprłtre, M. Figeac, A. Deste, and M.-C. Chartier-Harlin, "Involvement of the immune system, endocytosis and eIF2 signaling in both genetically determined and sporadic forms of Parkinsons disease", Neurobiology of Disease, vol. 63, pp. 165-170, Mar. 2014.

[8] J. Leitman, B. Barak, R. Benyair, M. Shenkman, U. Ashery, F. U. Hartl, and G. Z. Lederkremer, "ER stress-induced eIF $2 \alpha$ phosphorylation underlies sensitivity of striatal neurons to pathogenic huntingtin", PLoS ONE, vol. 9, no. 3, pp. e90803, Mar. 2014.

[9] C. R. Singh, B. Lee, T. Udagawa, S. S. Mohammad-Qureshi, Y. Yamamoto, G. D. Pavitt, and K. Asano, "An eIF5/eIF2 complex antagonizes guanine nucleotide exchange by eIF2B during translation initiation", The EMBO Journal, vol. 25, no. 19, pp. 4537-4546, oct 2006.

[10] M. D. Jennings, and G. D. Pavitt, "A new function and complexity for protein translation initiation factor eIF2B", Cell Cycle, vol. 13, no. 17 pp. 2660-2665, Sept. 2014

[11] T. Magazinnik, "Interplay between GCN2 and GCN4 expression, translation elongation factor 1 mutations and translational fidelity in yeast", Nucleic Acids Research, vol. 33, no. 14, pp. 4584-4592, Aug. 2005.

[12] B. A. Castilho, R. Shanmugam, R. C. Silva, R. Ramesh, B. M. Himme, and E. Sattlegger, "Keeping the eIF2 alpha kinase GCN2 in check", Biochimica et Biophysica Acta (BBA) - Molecular Cell Research, vol. 1843, no. 9, pp. 1948-1968, Sep. 2014.

[13] N. Donnelly, A. M. Gorman, S. Gupta, and A. Samali, "The eIF2 $\alpha$ kinases: Their structures and functions", Cellular and Molecular Life Sciences, vol. 70, no. 19 , pp. 3493-3511, Oct. 2013

[14] J. Dong, H. Qiu, M. Garcia-Barrio, J. Anderson, and A. G. Hinnebusch, "Uncharged tRNA activates GCN2 by displacing the protein kinase moiety from a bipartite tRNA-binding domain", Molecular Cell, vol 6, no. 2, pp. 269-279, Aug. 2000.

[15] M. F. Khan, S. Spurgeon, and T. von der Haar, "Origins of robustness in translational control via eukaryotic translation initiation factor (eIF) 2", Journal of Theoretical Biology, vol. 445, pp. 92-102, May 2018.

[16] J. Varner, J. Siddiqui, and S. Nayak, "Modelling and analysis of an ensemble of eukaryotic translation initiation models", IET Systems Biology, vol. 5, no. 1, pp. 2-14, Jan. 2011.

[17] M. F. Khan, S. K. Spurgeon, and X. G. Yan, "Modelling and dynamic behaviour of eIF2 dependent regulatory system with disturbances", IEEE Transactions on NanoBioscience, vol. 17, no. 4, pp. 518-524, 2018

[18] P. Sangthong, J. Hughes, and J. E. G. McCarthy, "Distributed control for recruitment, scanning and subunit joining steps of translation initiation", Nucleic Acids Research, vol. 35, no. 11, pp. 3573-3580, May 2007.

[19] J. Lequieu, A. Chakrabarti, S. Nayak, and J. D. Varner, "Computational modeling and analysis of insulin induced eukaryotic translation initiation”, PLoS Computational Biology, vol. 7, no. 11, pp. e1002263, Nov. 2011.

[20] H. Kitano, "Computational systems biology", Nature, vol. 420, no. 6912, pp. 206-210, Nov. 2002.

[21] C. Edwards, and S. Spurgeon, "Sliding mode control: Theory and applications", CRC Press, 1998.
[22] P. Erdi, and J. Toth, "Mathematical models of chemical reactions: Theory and applications of deterministic and stochastic models", Manchester University Press, 1989

[23] K. M. Hangos, and G. Szederknyi, "Mass action realizations of reaction kinetic system models on various time scales", Journal of Physics: Conference Series, vol. 268, p. 012009, Jan. 2011.

[24] J. E. Haag, A. V. Wouwer, and P. Bogaerts, "Dynamic modeling of complex biological systems: a link between metabolic and macroscopic description”, Mathematical Biosciences, vol. 193, no. 1, pp. 25-49, Jan. 2005.

[25] D. J. Wilkinson, "Stochastic modelling for quantitative description of heterogeneous biological systems", Nature Reviews Genetics, vol. 10, no. 2, pp. 122-133, Feb. 2009.

[26] T. You, G. M. Coghill, and A. J. P. Brown, "A quantitative model for mRNA translation in Saccharomyces cerevisiae", Yeast, vol. 27, no. 10, pp. 785-800, Mar. 2010.

[27] R. J. Dimelow, and S. J. Wilkinson, "Control of translation initiation: A model-based analysis from limited experimental data", Journal of The Royal Society Interface, vol. 6, no. 30, pp. 51-61, Jan. 2009.

[28] A. Laub, M. Heath, C. Paige, and R. Ward, "Computation of system balancing transformations and other applications of simultaneous diagonalization algorithms", IEEE Transactions on Automatic Control, vol. 32, no. 2, pp. 115-122, Feb. 1987.

[29] L. A. Segel, and M. Slemrod, "The quasi-steady-state assumption: A case study in perturbation", SIAM Review, vol. 31, no. 3, pp. 446-477, Jan. 1989.

[30] N. S. Bar, "Analysis of protein synthesis dynamic model in eukaryotic cells: Input control", Mathematical Biosciences, vol. 219, no. 2, pp. 8491, Jun. 2009.

[31] A. S. Spirin, "How does a scanning ribosomal particle move along the 5 '-untranslated region of eukaryotic mRNA? Brownian ratchet model, Biochemistry, vol. 48, no. 45, pp. 10688-10692, Nov. 2009.

[32] T. You, I. Stansfield, M. C. Romano, A. J. Brown, and G. M. Coghill, "Analysing GCN4 translational control in yeast by stochastic chemical kinetics modelling and simulation", BMC Systems Biology, vol. 5, no. 1, p. 131, 2011.

[33] K. L. Manchester, "Kinetic modelling of the effect of alpha subunit phosphorylation on the activity of the protein synthesis initiation factor eIF-2", Biochemistry International, vol. 22, no. 3, pp. 523-533, 1990.

[34] E. R. El-Haroun, D. P. Bureau, and J. P. Cant, "A mechanistic model of nutritional control of protein synthesis in animal tissues", Journal of Theoretical Biology, vol. 262, no. 2, pp. 361-369, Jan. 2010.

[35] K. Asano, T. Krishnamoorthy, L. Phan, G. D. Pavitt, and A. G. Hinnebusch, "Conserved bipartite motifs in yeast eIF5 and eIF2B epsilon, GTPase-activating and GDP-GTP exchange factors in translation initiation, mediate binding to their common substrate eIF2,", The EMBO Journal, vol. 18, no. 6, pp. 1673-1688, Mar. 1999.

[36] L. A. Hartwell, "Robust view of biochemical pathways", Nature, vol. 387, pp. 855-857, June 1997.

[37] L. Rong, and A. S. Perelson, "Modeling latently infected cell activation: Viral and latent reservoir persistence, and viral blips in HIV-infected patients on potent therapy", PLoS Computational Biology, vol. 5, no. 10, pp. e1000533, Oct. 2009.

[38] V. V. Ganusov, D. L. Barber, and R. J. De Boer, "Killing of targets by $\mathrm{CD} 8+\mathrm{T}$ cells in the mouse spleen follows the law of mass action", PLoS ONE, vol. 6, no. 1, pp. e15959, Jan. 2011.

[39] R. J. De Boer, D. Homann, and A. S. Perelson, "Different dynamics of $\mathrm{CD} 4+$ and $\mathrm{CD} 8+\mathrm{T}$ cell responses during and after acute lymphocytic choriomeningitis virus infection", The Journal of Immunology, vol. 171, no. 8, pp. 3928-3935, 2003.

[40] M. Hatch, "Vibration Simulation Using MATLAB and ANSYS", CRC Press, 2000 